03

\title{
Отражение света от слоя гиперболического метаматериала
}

\author{
(C) Н.С. Петров ${ }^{1}$, С.Н. Курилкина ${ }^{2}$, А.Б. Зимин ${ }^{3}$, В.Н. Белый ${ }^{2}$ \\ ${ }^{1}$ Институт повышения квалификации по новым направлениям развития техники, технологий \\ и экономики Белорусского национального технического университета, \\ 220107 Минск, Беларусь \\ ${ }^{2}$ Институт фризики им. Б.И. Степанова НАН Беларуси, \\ 220072 Минск, Беларусь \\ ${ }^{3}$ Белорусский государственный университет информатики и радиоэлектроники, \\ 220013 Минск, Беларусь \\ e-mail: s.kurilkina@ifanbel.bas-net.by
}

Поступила в редакцию 25.06.2019 г.

В окончательной редакции 25.06.2019 г.

Принята к публикации 12.07.2019 г.

Исследованы особенности отражения плоской эллиптически поляризованной электромагнитной волны, падающей из изотропной непоглощающей среды на плоско-параллельный слой гиперболического метаматериала (ГММ), оптическая ось которого параллельна границе раздела, а диагональные значения диэлектрической проницаемости меньше диэлектрической проницаемости изотропной среды. В зависимости от угла падения и угла между плоскостью падения и оптической осью ГММ возможны режимы, когда от границы раздела затухает или обыкновенная волна, или необыкновенная, или обе волны, причем в последнем случае при некоторых значениях углов затухание может быть неэкспоненциальным. Для этих трех режимов выполнены численные расчеты коэффициента отражения от слоя наноструктуры ITO/Ag. При изменении поляризации падающей волны энергетический коэффициент отражения изменяется от минимального до максимального значения, которые зависят от толщины слоя. В отличие от обычных анизотропных сред во всех случаях максимальное значение коэффициента отражения при увеличении толщины слоя стремится к единице. Когда амплитуда обыкновенной или необыкновенной волны не убывает от границы раздела, минимальный коэффициент отражения при увеличении толщины слоя вследствие интерференции периодически обращается в нуль. В случае, когда обе волны затухают, причем при некоторых условиях падения неэкспоненциально, минимальный коэффициент отражения обращается в нуль при некоторой толщине слоя, а затем стремится к единице.

Ключевые слова: метаматериал, анизотропная среда, однородная волна, неоднородная волна, отражение света.

DOI: $10.21883 /$ OS.2019.12.48691.225-19

\section{Введение}

В настоящее время внимание многих исследователей привлекают метаматериалы (ММ) - искусственно синтезированные среды, содержащие, как правило, металлические нановключения, упорядоченно внедренные в диэлектрическую матрицу и проявляющие электромагнитные свойства, не характерные для обычных изотропных и анизотропных сред $[1,2]$. Данный интерес связан с перспективами использования таких материалов для управления параметрами световых пучков, получения изображений с субволновым разрешением $[3,4]$, в нанолитографии ит.д. Описывающие данные среды усредненные (эффективные) проницаемости (диэлектрическая $\varepsilon$ и магнитная $\mu$ ) отличаются от таковых для их компонентов и существенно зависят от геометрических размеров нановключений и их взаимного расположения, что обусловливает возможность создания метаматериалов с заранее заданными уникальными оптическими свойствами [5].
Одним из видов ММ являются гиперболические метаматериалы (ГММ), для которых предполагается $\mu \approx 1$, а описывающий их одноосный тензор диэлектрической проницаемости $\varepsilon=\operatorname{diag}\left\{\varepsilon_{o}, \varepsilon_{o}, \varepsilon_{e}\right\}$ имеет главные значения проницаемостей (поперечной $\varepsilon_{o}$ и продольной $\left.\varepsilon_{e}\right)$, различающиеся знаком, следствием чего является появление гиперболической дисперсии [6]. Большинство практических применений ГММ (в частности, в сенсорике, ближнепольной микроскопии, в системах получения изображений) связано с особенностями формируемых в них электромагнитных волн, среди которых особое место занимают неоднородные волны, у которых плоскости равных фаз и равных амплитуд не параллельны между собой. Такие волны возникают в прозрачных средах при полном отражении света, при наклонном падении излучения в поглощающих (усиливающих) материалах. В работе [7] показана возможность существования на границе гиперболического метаматериала неоднородных волн особого типа, векторная амплитуда которых изменяется с глубиной проникновения волны в кристалл не по экспоненциальному, а более сложному закону. 


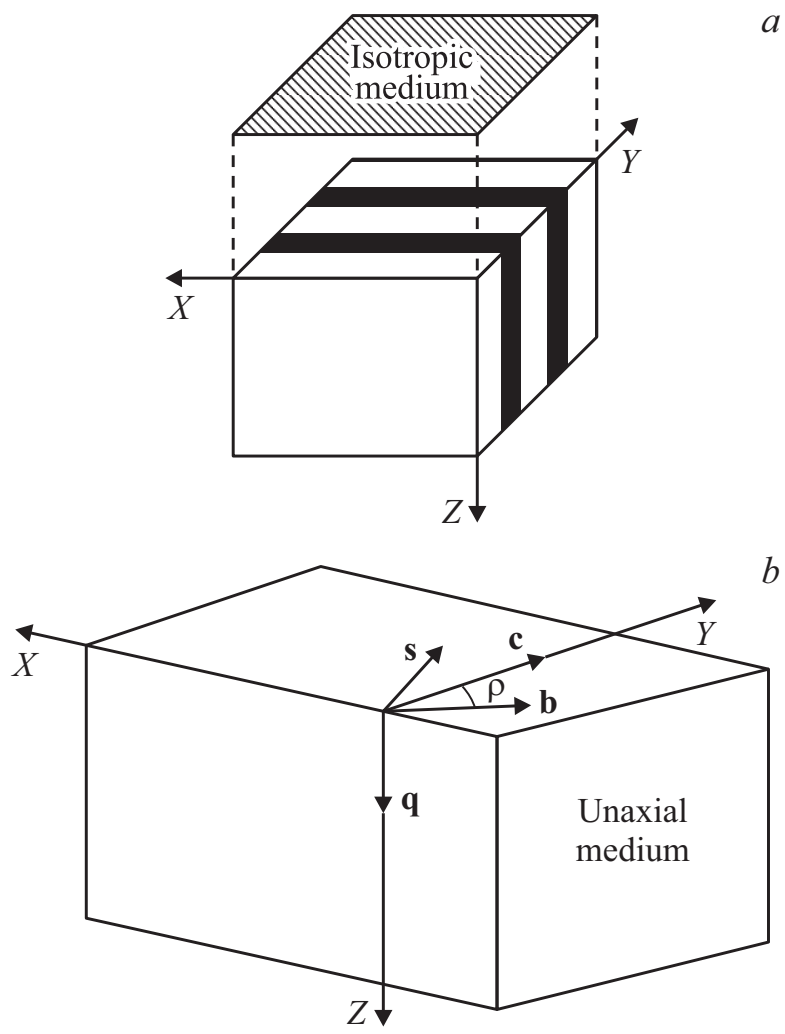

Рис. 1. Схема рассматриваемой структуры $(a)$ и ее представление в приближении эффективной среды $(b)$. c - единичный вектор вдоль оптической оси, $\mathbf{b}, \mathbf{q}, \mathbf{s}-$ тройка единичных векторов, где q - вектор, ортогональный границе раздела, $(\mathbf{c}, \mathbf{q})$ - главная плоскость анизотропной среды, $(\mathbf{b}, \mathbf{q})-$ плоскость падения световой волны, повернутая относительно главной плоскости на угол $\rho$.

В работе [8] рассмотрен случай отражения света слоем ГММ в условиях существования в нем этих особых неоднородных волн. В настоящей работе анализируется более общий случай отражения света от слоя гиперболического метаматериала в условиях слабого поглощения, когда в нем одновременно могут формироваться как однородная, так и неоднородная волны.

\section{Особенности отражения света слоем гиперболического метаматериала}

Пусть изотропная среда с диэлектрической проницаемостью $\varepsilon_{1}$ граничит со слоем гиперболического метаматериала, характеризуемого тензором диэлектрической проницаемости $\varepsilon=\operatorname{diag}\left\{\varepsilon_{o}, \varepsilon_{o}, \varepsilon_{e}\right\}=\varepsilon_{o}+\delta \mathbf{c} \cdot \mathbf{c}$, где $\delta=\varepsilon_{e}-\varepsilon_{o}, \mathbf{c}-$ единичный вектор вдоль оптической оси (точка между векторами обозначает их диадное произведение). В дальнейшем будем использовать систему координат, ось $z$ которой направлена внутрь ГММ, a ее начало совпадает с входной гранью слоя (рис. $1, a$ ).

Пусть из изотропного диэлектрика на границу раздела с плоскопараллельным слоем ГММ падает однородная эллиптически поляризованная электромагнитная волна. При решении поставленной задачи будем исходить из уравнений Максвелла

$$
\operatorname{rot} \mathbf{E}+\frac{1}{c} \frac{\partial \mathbf{B}}{\partial t}=0, \quad \operatorname{rot} \mathbf{H}-\frac{1}{c} \frac{\partial \mathbf{D}}{\partial t}=0,
$$

где $\mathbf{D}=\varepsilon \mathbf{E}, \mathbf{B} \cong \mathbf{H}$. Представим векторы электрической напряженности плоских волн, падающей из изотропного диэлектрика $\left(\mathbf{E}_{1}\right)$ и отраженной $\left(\mathbf{E}_{1}^{\prime}\right)$ слоем ГММ в следующем виде:

$$
\begin{aligned}
\mathbf{E}_{1} & =\left(A_{1} s+B_{1}\left[\mathbf{n}_{1} \mathbf{s}\right]\right) \exp \left(i \varphi_{1}\right) \\
& =\left(A_{1} s+B_{1} / n_{1}\left(-\xi \mathbf{q}+\eta_{1} \mathbf{b}\right)\right) \exp \left(i \varphi_{1}\right), \\
\mathbf{E}_{1}^{\prime} & \left.=\left(A_{1}^{\prime} s+B_{1}^{\prime}\left[\mathbf{n}_{1}^{\prime} \mathbf{s}\right]\right)\right) \exp \left(i \varphi_{1}^{\prime}\right) \\
& =\left(A_{1}^{\prime} s+B_{1}^{\prime} / n_{1}\left(-\xi \mathbf{q}-\eta_{1} \mathbf{b}\right)\right) \exp \left(i \varphi_{1}\right) .
\end{aligned}
$$

Здесь фазовый множитель $e^{-i \omega t}$ опущен; $A_{1}\left(B_{1}\right)$ и $A_{1}^{\prime}\left(B_{1}^{\prime}\right)$ - соответственно амплитуды волн с векторами поляризации, ориентированными перпендикулярно и параллельно плоскости падения, $\varphi_{1}=k \mathbf{m}_{1} \mathbf{r}, \varphi_{1}^{\prime}=k \mathbf{m}_{1}^{\prime} \mathbf{r}$ $(\mathbf{r}-$ радиус-вектор $), \mathbf{m}_{1}$ и $\mathbf{m}_{1}^{\prime}-$ так называемые векторы рефракции этих волн, представимые в виде

$$
\mathbf{m}_{1}=n_{1} \mathbf{n}_{1}=\xi \mathbf{b}+\eta_{1} \mathbf{q}, \quad \mathbf{m}_{1}^{\prime}=n_{1} \mathbf{n}_{1}^{\prime}=\xi \mathbf{b}-\eta_{1} \mathbf{q} .
$$

При этом для них выполняются соотношения

$$
\mathbf{m}_{1}^{2}=\left(\mathbf{m}_{1}^{\prime}\right)^{2}=n_{1}^{2}=\varepsilon_{1}=\xi^{2}+\eta_{1}^{2},
$$

где $n_{1}=\sqrt{\varepsilon_{1}}-$ показатель преломления изотропной среды, из которой падает излучение, $\mathbf{n}_{1}$ и $\mathbf{n}_{1}^{\prime}-$ единичные векторы в направлении распространения фазы волны, $\xi=n_{1} \sin \alpha, \eta_{1}=n_{1} \cos \alpha, \alpha-$ угол падения, изменяющийся в интервале $0 \leq \alpha \leq \pi / 2\left(0 \leq \xi^{2} \leq \varepsilon_{1}\right)$, $\mathbf{b}, \mathbf{q}$ и $\mathbf{s}-$ тройка взаимно перпендикулярных ортов (b - вдоль границы раздела сред, $\mathbf{q}$ - нормаль к границе ГММ, направленная вглубь слоя, $\mathbf{s}-$ нормаль к плоскости падения $(s=[\mathbf{b q}])$ (рис. $1, b)$.

Векторы напряженности магнитного поля Н также определяются из уравнений (1), откуда для случая плоских волн следует $\mathbf{H}=[\mathbf{m E}]$. Тогда

$$
\begin{aligned}
\mathbf{H}_{1} & =\left(A_{1}\left[\mathbf{m}_{1} \mathbf{s}\right]-n_{1} B_{1} \mathbf{s}\right) \exp \left(i \varphi_{1}\right) \\
& =\left(A_{1}\left(-\xi \mathbf{q}+\eta_{1} \mathbf{b}\right)-n_{1} B_{1} \mathbf{s}\right) \exp \left(i \varphi_{1}\right), \\
\mathbf{H}_{1}^{\prime} & =\left(A_{1}^{\prime}\left[\mathbf{m}_{1}^{\prime} \mathbf{s}\right]-n_{1} B_{1}^{\prime} \mathbf{s}\right) \exp \left(i \varphi_{1}^{\prime}\right) \\
& =\left(A_{1}^{\prime}\left(-\xi \mathbf{q}-\eta_{1} \mathbf{b}\right)-n_{1} B_{1}^{\prime} \mathbf{s}\right) \exp \left(i \varphi_{1}\right) .
\end{aligned}
$$

Векторы электрического и магнитного поля волны, прошедшей анизотропный слой, представим в виде, аналогичном выражениям для $\mathbf{E}_{1}\left(\mathbf{E}_{1}^{\prime}\right)$ и $\mathbf{H}_{1}\left(\mathbf{H}_{1}^{\prime}\right)$, а именно

$$
\begin{aligned}
\mathbf{E}_{2} & =\left(A_{2} s+B_{2}\left[n_{2} \mathbf{s}\right]\right) \exp \left(i \varphi_{2}\right) \\
& =\left(A_{2} s+B_{2} / n_{2}\left(-\xi \mathbf{q}+\eta_{2} \mathbf{b}\right)\right) \exp \left(i \varphi_{2}\right),
\end{aligned}
$$




$$
\begin{aligned}
\mathbf{H}_{2} & =\left(A_{2}\left[\mathbf{m}_{2} s\right]-n_{2} B_{2} \mathbf{s}\right) \exp \left(i \varphi_{2}\right) \\
& =\left(A_{2}\left(-\xi \mathbf{q}+\eta_{2} \mathbf{b}\right)+n_{2} B_{2} \mathbf{s}\right) \exp \left(i \varphi_{2}\right),
\end{aligned}
$$

где $\quad \mathbf{m}_{2}=\xi \mathbf{b}+\eta_{2} \mathbf{q}, \quad \eta_{2}=\sqrt{\mathbf{m}_{2}^{2}-\xi^{2}}, \quad\left(\mathbf{m}_{2}^{2}=\varepsilon_{2}=n_{2}^{2}\right)$, $\varphi_{2}=k \mathbf{m}_{2} \mathbf{r}, n_{2}-$ показатель преломления изотропной среды, граничащей со слоем гиперболического метаматериала с другой его стороны. Далее принято $n_{2}=n_{1}$, $\left(\eta_{2}=\eta_{1}\right)$, т.е. окружающие анизотропный слой изотропные среды одинаковы.

В слое гиперболического метаматериала распространяются две пары встречных волн, для которых векторы электрической и магнитной напряженностей получаются как решения уравнений Максвелла и представляются следующим образом:

$$
\begin{gathered}
\mathbf{E}=C_{1} \mathbf{E}_{o} \exp \left(i k \mathbf{m}_{o} \mathbf{r}\right)+C_{1}^{\prime} \mathbf{E}_{0}^{\prime} \exp \left(i k \mathbf{m}_{0}^{\prime} \mathbf{r}\right) \\
+C_{2} \mathbf{E}_{e} \exp \left(i k \mathbf{m}_{e} \mathbf{r}\right)+C_{2}^{\prime} \mathbf{E}_{e}^{\prime} \exp \left(i k \mathbf{m}_{e}^{\prime} \mathbf{r}\right), \\
\mathbf{H}=C_{1} \mathbf{H}_{o} \exp \left(i k \mathbf{m}_{o} \mathbf{r}\right)+C_{1}^{\prime} \mathbf{H}_{o}^{\prime} \exp \left(i k \mathbf{m}_{o}^{\prime} \mathbf{r}\right) \\
+C_{2} \mathbf{H}_{e} \exp \left(i k \mathbf{m}_{e} \mathbf{r}\right)+C_{2}^{\prime} \mathbf{H}_{e}^{\prime} \exp \left(i k \mathbf{m}_{e}^{\prime} \mathbf{r}\right), \\
\mathbf{E}_{o}=-u \gamma_{1} \mathbf{s}+v \gamma_{1} \mathbf{b}+i \xi v \mathbf{q}, \\
\mathbf{E}_{o}^{\prime}=u \gamma_{1} \mathbf{s}-v \gamma_{1} \mathbf{b}+i \xi v \mathbf{q}, \\
\mathbf{H}_{o}=i v \varepsilon_{o} \mathbf{s}-i u \gamma_{1}^{2} \mathbf{b}+u \xi \gamma_{1} \mathbf{q}=\left[\mathbf{m}_{o} \mathbf{E}_{o}\right], \\
\mathbf{H}_{o}^{\prime}=i v \varepsilon_{o} \mathbf{s}-i u \gamma_{1}^{2} \mathbf{b}-u \xi \gamma_{1} \mathbf{q}=\left[\mathbf{m}_{o}^{\prime} \mathbf{E}_{o}^{\prime}\right], \\
\mathbf{E}_{e}=u \gamma_{1}^{2} \mathbf{b}-v \varepsilon_{o} \mathbf{s}+i u \xi \gamma_{2} \mathbf{q}, \\
\mathbf{E}_{e}^{\prime}=u \gamma_{1}^{2} \mathbf{b}-v \varepsilon_{o} \mathbf{s}-i u \xi \gamma_{2} \mathbf{q}, \\
\mathbf{H}_{e}=\varepsilon_{o}\left(-i v \gamma_{2} \mathbf{b}+i u \gamma_{2} \mathbf{s}+v \xi \mathbf{q}\right), \\
\mathbf{H}_{e}^{\prime}=\varepsilon_{o}\left(i v \gamma_{2} \mathbf{b}-i u \gamma_{2} \mathbf{s}+v \xi \mathbf{q}\right) .
\end{gathered}
$$

Здесь $\quad \mathbf{m}_{o}=\xi \mathbf{b}+i \gamma_{o} \mathbf{q}, \quad m_{o}^{\prime}=\xi \mathbf{b}-i \gamma_{o} \mathbf{q}, \quad \gamma_{1}=\gamma_{o}=$ $=\sqrt{\xi^{2}-\varepsilon_{o}},\left(\mathbf{m}_{o}^{2}=\varepsilon_{o}\right), \mathbf{m}_{e}=\xi \mathbf{b}+i \gamma_{e} \mathbf{q}, \mathbf{m}_{e}^{\prime}=\xi \mathbf{b}-i \gamma_{e} \mathbf{q}$, $\gamma_{2}=\gamma_{e}=\sqrt{\xi^{2}\left(\varepsilon_{0} \sin ^{2} \rho+\varepsilon_{e} \cos ^{2} \rho\right) / \varepsilon_{0}-\varepsilon_{e}}=$

$=\sqrt{\xi^{2}-\varepsilon_{0}+\left(\varepsilon_{e}-\varepsilon_{o}\right)\left(\xi^{2} \cos ^{2} \rho / \varepsilon_{o}-1\right)}, \quad \mathbf{c}=\cos \rho \mathbf{b}+$ $+\sin \rho \mathbf{s}=u \mathbf{b}+v \mathbf{s}, k=\omega / c$.

Отметим, что при $\xi^{2} \cos ^{2} \rho / \varepsilon_{o}=1$ двойное лучепреломление в среде отсутствует, и возникает особая волна, проанализированная ранее в [8]. В этом случае для нахождения решений вместо системы (8) следует использовать результаты [8]. В неособом случае из стандартных граничных условий (равенства на границе раздела всех компонент векторов магнитной напряженности и тангенциальных b компонент векторов электрической напряженности) получается система уравнений для нахождения восьми скалярных амплитуд $A_{1}^{\prime}\left(B_{1}^{\prime}\right)$, $C_{1}\left(C_{1}^{\prime}\right), C_{2}\left(C_{2}^{\prime}\right), A_{2}, B_{2}$ волн (2a), (2b), (5a), (5b), (6a), (6b), (7a), (7b):

$$
\begin{gathered}
\eta_{1}\left(A_{1}-A_{1}^{\prime}\right)+i u \gamma_{1}^{2}\left(C_{1}+C_{1}^{\prime}\right)+i v \gamma_{2} \varepsilon_{o}\left(C_{2}-C_{2}^{\prime}\right)=0, \\
A_{1}+A_{1}^{\prime}+u \gamma_{1}\left(C_{1}-C_{1}^{\prime}\right)+v \varepsilon_{o}\left(C_{2}+C_{2}^{\prime}\right)=0, \\
n_{1}\left(B_{1}+B_{1}^{\prime}\right)+i v \varepsilon_{o}\left(C_{1}+C_{1}^{\prime}\right)+i u \gamma_{2} \varepsilon_{o}\left(C_{2}-C_{2}^{\prime}\right)=0,
\end{gathered}
$$

$$
\begin{gathered}
\eta_{1} / n_{1}\left(-B_{1}+B_{1}^{\prime}\right)+v \gamma_{1}\left(C_{1}-C_{1}^{\prime}\right)+u \gamma_{1}^{2}\left(C_{2}+C_{2}^{\prime}\right)=0, \\
\eta_{1} A_{2} \exp (i \psi)+i u \gamma_{1}^{2}\left(C_{1 \vartheta}+C_{1 \vartheta}^{\prime}\right)+i v \gamma_{2} \varepsilon_{o}\left(C_{2 \vartheta}-C_{2 \vartheta}^{\prime}\right)=0, \\
A_{2} \exp (i \psi)+u \gamma_{1}\left(C_{1 \vartheta}-C_{1 \vartheta}^{\prime}\right)+v \varepsilon_{o}\left(C_{2 \vartheta}+C_{2 \vartheta}^{\prime}\right)=0, \\
n_{1} B_{2} \exp (i \psi)+i v \varepsilon_{o}\left(C_{1 \vartheta}+C_{1 \vartheta}^{\prime}\right)+i u \gamma_{2} \varepsilon_{o}\left(C_{2 \vartheta}-C_{2 \vartheta}^{\prime}\right)=0, \\
-\eta_{1} / n_{1} B_{2} \exp (i \psi)+v \gamma_{1}\left(C_{1 \vartheta}-C_{1 \vartheta}^{\prime}\right)+u \gamma_{1}^{2}\left(C_{2 \vartheta}+C_{2 \vartheta}^{\prime}\right)=0 .
\end{gathered}
$$

Здесь $\xi=k h, h-$ толщина слоя, $\vartheta_{1}=\gamma_{1} \xi^{\prime}, \vartheta_{2}=\gamma_{2} \zeta^{\prime}$, $\psi=\eta_{1} \xi^{\prime}, n=\sqrt{\varepsilon_{o}}, C_{1 \vartheta}=C_{1} \exp \left(-\vartheta_{1}\right), C_{2 \vartheta}=C_{2} \exp \left(\vartheta_{2}\right)$, $C_{1 \vartheta}^{\prime}=C_{1}^{\prime} \exp \left(\vartheta_{1}\right), C_{2 \vartheta}^{\prime}=C_{2}^{\prime} \exp \left(\vartheta_{2}\right)$. Аналитическое решение данной системы уравнений, определяющей векторы электрической и магнитной напряженностей волн, отраженных слоем ГММ и прошедших через него, и, следовательно, энергетические коэффициенты отражения и пропускания, достаточно громоздко. Однако эта система может быть решена численными методами для произвольной, в общем случае эллиптической, поляризации падающего света.

\section{Результаты расчетов коэффициента отражения от слоя гиперболического метаматериала}

Пусть гиперболический метаматериал сформирован на основе слоисто-периодической металлодиэлектрической структуры. В приближении эффективной среды, когда толщина каждого входящего в структуру слоя достаточно мала, т.е. $\left|k_{d} d_{d}\right| \ll 1,\left|k_{m} d_{m}\right| \ll 1$, где $k_{d}$, $k_{m}$ - соответственно волновые числа диэлектрического и металлического слоев, многослойная структура может рассматриваться как эффективная оптически одноосная среда. При этом тензор диэлектрической проницаемости ее имеет собственные значения, определяемые выражениями

$$
\varepsilon_{o}=(1-f) \varepsilon_{d}+f \varepsilon_{m}, \quad \varepsilon_{e}=\left[\frac{1-f}{\varepsilon_{d}}+\frac{f}{\varepsilon_{m}}\right]^{-1} .
$$

Здесь $f=d_{m} /\left(d_{m}+d_{d}\right)$ - фактор заполнения (объемная доля, занимаемая в структуре металлом), $\varepsilon_{d}, \varepsilon_{m}-$ соответственно проницаемости диэлектрического и металлического слоев. При этом величина $\varepsilon_{m}$ описывается формулой Друде:

$$
\begin{aligned}
\varepsilon_{m}(\omega) & =\varepsilon_{\infty}-\omega_{p}^{2} /\left(\omega^{2}+i \omega \Gamma\right) \\
& =\varepsilon_{\infty}-\omega_{p}^{2} /\left(\omega^{2}+\Gamma^{2}\right)+i \omega_{p}^{2} \Gamma /\left[\omega\left(\omega^{2}+\Gamma^{2}\right)\right]
\end{aligned}
$$

где $\omega_{p}-$ плазменная частота, $\varepsilon_{\infty}-$ постоянная, описывающая вклад межзонных переходов, $\Gamma=V_{F} / l-$ постоянная затухания, $V_{F}-$ скорость Ферми, $l-$ среднее значение свободного пути электрона в объемном металле. Для серебра, например, $\varepsilon_{\infty}=5, \omega_{p}=14 \cdot 10^{15} \mathrm{~s}^{-1}$, $\Gamma=32 \cdot 10^{12} \mathrm{~s}^{-1}, V_{F}=1.4 \cdot 10^{6} \mathrm{~ms}^{-1}[1]$. Расчет, согласно выражению (10) для слоисто-периодической среды 


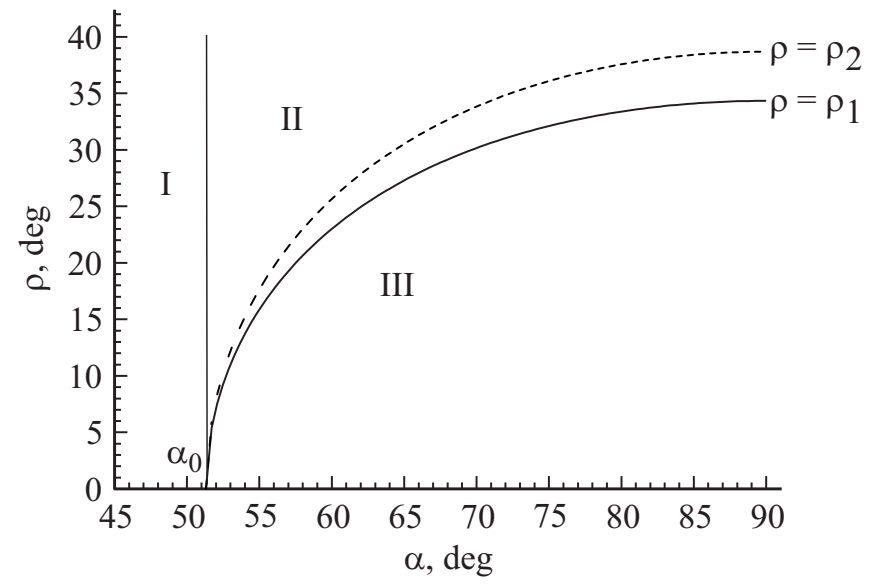

Рис. 2. Зависимости от угла падения света $\alpha$ предельного угла $\rho_{1}$ между плоскостью падения и оптической осью ГММ, при котором выполняется условие полного отражения для необыкновенной волны (сплошная кривая), а также угла $\rho_{2}$, для которого внутри слоя метаматериала отсутствует двойное лучепреломление (штриховая кривая). ГММ сформирован на основе слоисто-периодической среды ITO $/ \mathrm{Ag}\left(d_{m}=20 \mathrm{~nm}, f=0.3\right)$, со слоем граничит оптическое стекло ВК7. $\lambda=360 \mathrm{~nm}$.

$\mathrm{ITO} / \mathrm{Ag}\left(d_{m}=20 \mathrm{~nm}, f=0.3\right)$, при длине падающей световой волны $360 \mathrm{~nm}$ дает значения $\varepsilon_{o}=1.44, \varepsilon_{e}=-6.32$. Для граничащих со слоем изотропных сред (например, оптическое стекло ВК7) $\varepsilon_{1}=n_{1}^{2}=2.36$.

На рис. 2 представлены рассчитанные для структуры „ВК-7 - слой ITO/Ag - ВК-7““зависимости, устанавливающие связь между углом падения $\alpha$ световой волны на слой ГММ и следующими интересующими нас предельными значениями углов $\rho$ между плоскостью падения и оптической осью: $\rho_{1}$, определяющим переход к режиму полного отражения для необыкновенной волны внутри слоя ГММ $\left(\gamma_{2}=0\right)$, и $\rho_{2}-$ углом, для которого внутри слоя метаматериала отсутствует двойное лучепреломление $\left(\gamma_{1}=\gamma_{2}, \gamma_{1}>0\right)$.

Как видно из рис. 2 , можно выделить три различных режима отражения света от слоя ГММ, разделенных сплошными кривыми. Для первого из них (область I, соответствующая случаю $\alpha<\alpha_{0}=51.3^{\circ}$ ) в слое независимо от угла между плоскостью падения и оптической осью метаматериала распространяются однородная обыкновенная волна и неоднородная необыкновенная. Для второго (область II, соответствующая случаю $\alpha>\alpha_{0}$ и $\left.\rho>\rho_{1}\right)-$ существующие в слое обыкновенная и необыкновенная волны являются неоднородными. Для третьего режима (область III, соответствующая случаю $\alpha>\alpha_{0}$ и $\rho<\rho_{1}$ ) необыкновенная волна в слое является однородной, а обыкновенная неоднородной. Например, для $\alpha=53^{\circ}$ второй (третий) режим отражения реализуется при $\rho>11^{\circ}\left(\rho<11^{\circ}\right)$, а двойное лучепреломление отсутствует (т.е. в слое возникает неоднородная волна особого типа, для которой имеет место неэкспоненциальное уменьшение амплитуды при удалении от границы слоя [7]) при $\rho=12.2^{\circ}$.

Для вычисления энергетического коэффициента отражения была вычислена двумерная матрица амплитудного коэффициента отражения, преобразующая коэффициенты $A_{1}, B_{1}$ в коэффициенты $A_{1}^{\prime}, B_{1}^{\prime}$. Двумерная матрица энергетического коэффициента отражения получается умножением матрицы амплитудного коэффициента отражения на свою эрмитово сопряженную матрицу. При заданных углах $\alpha$ и $\rho$ энергетический коэффициент отражения $R=\left|\mathbf{E}_{1}^{\prime}\right|^{2} /\left|\mathbf{E}_{1}\right|^{2}$ при изменении поляризации падающей волны изменяется от максимального $R_{\max }$ до минимального $R_{\min }$ значения, которые вычисляются как собственные значения матрицы энергетического коэффициента отражения. Состояния поляризации падающей волны, при которых достигаются значения $R_{\max }$ и $R_{\min }$, в общем случае являются эллиптическими с ортогональными осями эллипсов [9]. Направления осей эллипсов зависят от $\alpha, \rho, h$ и других параметров. На рис. 3 представлена зависимость $R_{\max }$ и $R_{\min }$ от толщины слоя ГММ для значений углов $\alpha$ и $\rho$, соответствующих различным режимам отражения, представленным на рис. 2. Как видно из рис. $3, a$ и $3, b$, в случае, если лишь одна из волн (обыкновенная или необыкновенная) в слое является неоднородной и экспоненциально убывает от границы раздела, минимальное значение коэффициента отражения периодически меняется с толщиной слоя от нуля до некоторого значения, меньшего единицы, а максимальное значение быстро увеличивается и уже при малых $h \cong 200 \mathrm{~nm}$ оказывается близким к единице. Минимумы зависимости $R_{\min }(h)$ для случая существования в ГММ однородной обыкновенной волны (рис. $3, a$ ) достаточно широкие и соответствуют условию ее интерференционного пропускания. В случае существования в слое ГММ однородной необыкновенной волны (рис. $3, b)$ минимумы зависимости $R_{\min }(h)$ являются более узкими и также соответствуют условиям интерференционного пропускания.

Представляет интерес случай, когда углы $\rho$ и $\alpha$ расположены в области II. Здесь возможно выполнение равенства $\rho=\rho_{2}$, когда возникает особое решение уравнений Максвелла, рассмотренное в работе [7]. При всех значениях $\rho>\rho_{1}\left(\rho_{2}>\rho>\rho_{1}-\right.$ рис. $3, c, \rho>\rho_{2}-$ pис. $3, d$ ), в том числе и при $\rho=\rho_{2}$ (рис. $3, e$ ), коэффициент отражения $R_{\min }(h)$ при увеличении толщины слоя вырастает до некоторого максимального значения и становится равным нулю при некоторой толщине слоя $h_{c}<\lambda$, зависящей от углов $\rho$ и $\alpha$. При дальнейшем увеличении толщины он увеличивается и стремится к единице в отличие от интерференционного поведения в областях I и III. При этом в случае особого решения при $\rho=\rho_{2}$ система (9) вырождается до четырех уравнений, и корректные значения для полей в слое и коэффициента отражения получаются из решений, приведенных в [7]. Обращение коэффициента отражения от слоя в нуль при возбуждении в нем экспоненциально затухающих волн имеет место только для ГММ с определенными 

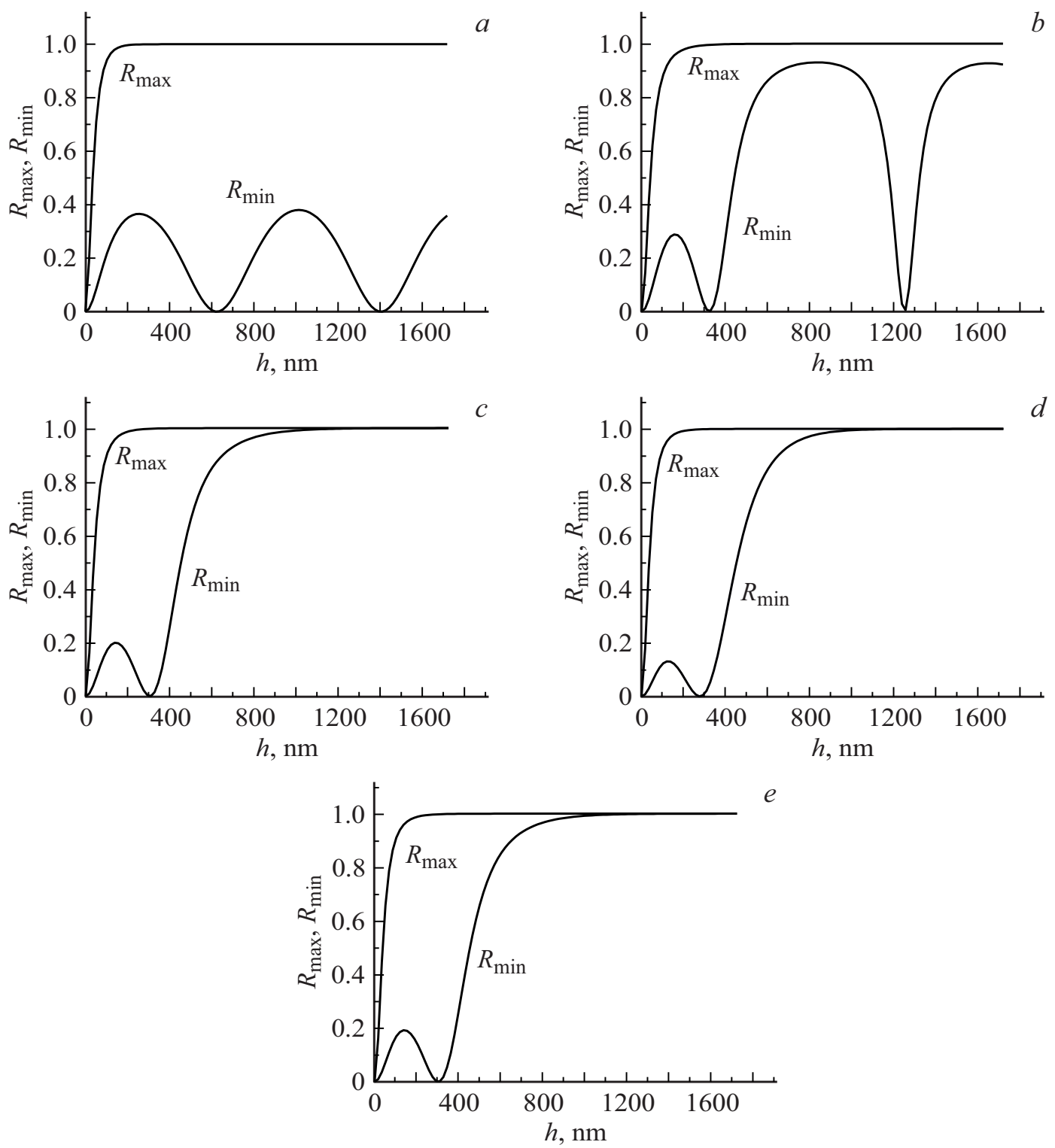

Рис. 3. Зависимости величин максимального $R_{\max }$ и минимального $R_{\min }$ коэффициентов отражения, полученных при измерении отражения линейно-поляризованного света всевозможных состояний поляризации, от толщины слоя ГММ на основе слоистопериодической среды ITO/Ag $\left(d_{m}=20 \mathrm{~nm}, f=0.3\right):(a) \alpha=50^{\circ}, \rho=12^{\circ},(b) \alpha=53^{\circ}, \rho=10^{\circ},(c) \alpha=53^{\circ}, \rho=12^{\circ},(d) \alpha=53^{\circ}$, $\rho=14^{\circ},(e) \alpha=53^{\circ}, \rho=12.2^{\circ}$. В случае $(e)$ двойное лучепреломление отсутствует. Со слоем граничит оптическое стекло ВК7. $\lambda=360 \mathrm{~nm}$.

параметрами и не возникает, например, в случае полного отражения от анизотропной среды с $\varepsilon_{o}, \varepsilon_{e}>0$, когда волны в слое экспоненциально убывают.

\section{Заключение}

Таким образом, в настоящей работе исследованы особенности отражения света от слоя гиперболического метаматериала в случае, когда в нем возможно существование как одной неоднородной волны (обыкновенной или необыкновенной), так и обеих. Получены выражения, позволяющие рассчитать коэффициенты отражения излучения при произвольной поляризации падающего света. Выполнены численные расчеты коэффициента отражения от слоя наноструктуры ITO/Ag для различных режимов отражения. Показано, что если в слое ГММ существует только одна (обыкновенная или необыкновенная) неоднородная волна, то коэффициент отражения $R_{\min }$ при определенных толщинах слоя обращается в нуль, что соответствует условию интерференционного пропускания. При возникновении в слое экспоненциально убывающих волн, в том числе при возникновении при определенных углах падения особой неоднородной волны, характеризуемой неэкспоненциальным уменьшением амплитуды при удалении от границы слоя, в зависимости от толщины слоя минимального значения коэффициента 
отражения, рассчитанного для всевозможных значений поляризации падающего света, появляется минимум, соответствующий полному пропусканию света.

Полученные результаты имеют перспективы применения в микроскопии, а также при разработке новых методов зондирования приповерхностных дефектов различных материалов.

\section{Конфликт интересов}

Авторы заявляют, что у них нет конфликта интересов.

\section{Список литературы}

[1] Cai W., Shalaev V. Optical Metamaterials: Fundamentals and Applications. Springer, 2010. doi 10.1007/978-1-4419-1151-3

[2] Metamaterials Handbook 1: Theory and Phenomena of Metamaterials. CRC Press, 2009.

[3] Pendry J.B. // Phys. Rev. Lett. 2000. V. 85. P. 3966. doi 10.1103/PhysRevLett.85.3966

[4] Fang N., Lee H., Sun C., Zhang X. // Science. 2005. V. 308. P. 534. doi 10.1126/science.1108759

[5] Kidwai O., Zhukovsky S.V., Sipe J.E. // Phys. Rev. 2012. V. A 85. P. 053842. doi 10.1103/PhysRevA.85.053842

[6] Shekhar P., Atkinson J., Jacob Z. // Nano Convergence. 2014. V. 1. P. 14. doi 10.1186/s40580-014-0014-6

[7] Kurilkina S.N., Petrov N.S., Zimin A.B., Belyi V.N. // J. Opt. 2017. V. 19. P. 125102. doi 10.1088/2040-8986/aa945c

[8] Петров Н.С., Курилкина С.Н., Зимин А.Б., Белый В.Н. // Опт. и спектр. 2019. Т. 126. С. 319. doi 10.21883/OS.2019.03.47373.311-18

[9] Федоров Ф.И. Теория гиротропии. Минск, 1976. 